\title{
De la Universidad al mercado de trabajo: el caso de los egresados de la Universidad Michoacana
}

Zalapa Lúa, Elsa Edith; Silva Riquer, Jorge; Favila Tello, Antonio

De la Universidad al mercado de trabajo: el caso de los egresados de la Universidad Michoacana

Revista Educación, vol. 43, núm. 2, 2019

Universidad de Costa Rica, Costa Rica

Disponible en: http://www.redalyc.org/articulo.oa?id=44058158015

DOI: https://doi.org/10.15517/revedu.v43i2.31713

Esta obra está bajo una Licencia Creative Commons Atribución-NoComercial-SinDerivar 3.0 Internacional. 


\title{
De la Universidad al mercado de trabajo: el caso de los egresados de la Universidad Michoacana
}

\author{
From the University to the Job Market: Graduates from Michoacana University (Mexico)
}

Elsa Edith Zalapa Lúa

Universidad Michoacana de San Nicolás de Hidalgo,

México

elsa.zalapa.lua@gmail.com

(iD http://orcid.org/0000-0003-2280-4483

Jorge Silva Riquer

Universidad Michoacana de San Nicolás de Hidalgo,

México

jsriquer@gmail.com

(D) http://orcid.org/0000-0003-1626-3297

Antonio Favila Tello

Universidad Michoacana de San Nicolás de Hidalgo,

México

antonio_favila@hotmail.com

(iD http://orcid.org/0000-0001-8652-147X

\author{
DOI: https://doi.org/10.15517/revedu.v43i2.31713 \\ Redalyc: http://www.redalyc.org/articulo.oa?id=44058158015
}

Recepción: 09 Diciembre 2017

Aprobación: 15 Mayo 2019

\section{Resumen:}

La inserción laboral es un indicador altamente valorado tanto en los estudios de egresados, como en las acreditaciones a las cuales se someten las Instituciones de Educación Superior (IES). El objetivo de esta investigación fue analizar tres dimensiones de la encuesta de egresados que se aplica en la Universidad Michoacana de San Nicolás de Hidalgo (UMSNH) para dar cuenta sobre cómo es el tránsito de las personas egresadas una vez que concluyen sus estudios, hasta conseguir, o no, un empleo. El método es cuantitativo, el diseño es no experimental y de alcance descriptivo. En el estudio participaron 11.444 egresados solo del nivel superior. Un $44,5 \%$ reportó no tener empleo al momento de la aplicación y un 50,2\% detalló que sí. La principal dificultad para conseguir empleo fue no estar titulado (30,2\%), lo que influyó para conseguirlo fue la coincidencia en la formación con las necesidades de la empresa (80\%). El ingreso mejoró para todos los campos formativos una vez que egresaron. La mayoría de los que laboran están ubicados en microempresas. En general, los egresados de esta universidad reportan porcentajes altos de satisfacción laboral, siendo el salario y la posición jerárquica alcanzada los aspectos en donde reportan menos satisfacción laboral. De los resultados de este tipo de estudios, se pueden establecer líneas de trabajo entre IES y representantes de la estructura productiva quienes tienen injerencia directa en la configuración de los mercados de trabajo. Ello con la finalidad de influir en el desarrollo al menos del contexto inmediato.

Palabras Clave: Egresados, Educación superior, Mercado de trabajo, Campos formativos.

\section{Abstract:}

Job placement is highly regarded indicator among graduates, as is attaining accreditation among higher education institutions. This study analyzes three dimensions of a survey conducted among graduates of the University of San Nicolás Hidalgo (UMSNH) in Michoacan, Mexico. The survey objective was to learn what jobs graduates obtained, if any, upon graduation. This was a quantitative and descriptive study with a non-experimental design. Approximately 11,444 graduates were surveyed, $44.5 \%$ of which reported being unemployed while $50.2 \%$ were employed. It was determined that the main deterrent to securing a job was not having a college degree $(30.2 \%)$ and the main asset to finding a job was matching the subject studies at university with the needs of the company (80\%). Most graduates were employed by small businesses. Overall, UMSNH graduates report high job satisfaction, stating that their salary and hierarchical position represented was what caused the least job satisfaction. One can 
conclude from this type of study that universities and employers can work together to help university students enter the workforce upon graduation and help improve the current employer scenario.

KEYWORDs: Graduates, Higher education, Training fields, Formative fields.

\section{INTRODUCCIÓN}

La Universidad Michoacán de San Nicolás de Hidalgo (UMSNH) es una institución pública y laica, en este año festejó su cumpleaños número cien. En la actualidad, a nivel nacional se encuentra entre las diez primeras universidades públicas de México, según el Ranking QS (2017). Esta institución se ubica en la ciudad de Morelia en el estado de Michoacán. Si bien cuenta con nodos descentralizados de la capital para ofrecer sus servicios, se puede decir que la mayoría de la matrícula escolar está concentrada en la capital del estado. Una característica de la UMSNH es que año con año, ingresan estudiantes principalmente de estados vecinos, que por diversas causas no pudieron acceder al nivel superior en las universidades públicas de sus localidades. Dentro de la oferta educativa, se imparte educación del nivel medio superior, nivel superior ofrece 38 diferentes licenciaturas, 10 especialidades, 35 maestrías y 24 doctorados y para el ciclo 2017-2018 contaba con 55,000 estudiantes.

En México la tendencia es que por cada 100 estudiantes que inician su educación primaria, aproximadamente solo 21 llegarán a inscribirse en una carrera universitaria y 13 obtendrán un título de educación superior (Secretaría de Educación Pública, 2017), tendencia que es similar a nivel estatal. Este panorama se complejiza al incluir en el análisis, el porcentaje de personas egresadas que logran obtener un empleo acorde a su formación profesional. Pues si bien no corresponde a las universidades o no está en sus manos el cambiar la estructura productiva, que configura el mercado de trabajo [MT] de las regiones, sí es un deber velar porque sus egresados salgan con buenas bases teóricas y prácticas, es decir que las universidades tienen que ser pertinentes no solo a nivel educativo, sino también deben ser pertinentes a nivel social. Esto sustentado bajo la premisa de que, si bien la educación no garantiza el obtener un empleo, si reduce el riesgo de desempleo en la mayoría de los países y permite acceder a mejores niveles salariales (Asociación Nacional de Universidades e Instituciones de Educación Superior [ANUIES], 2000) así como contribuye a una mayor productividad siempre y cuando existan las oportunidades laborales para los egresados.

Para obtener información al respecto se elaboran diversos tipos de estudios, entre ellos los de egresados y los de MT. En el caso de México, los primeros suelen efectuarse, siguiendo los lineamientos propuestos en el esquema básico para estudios de egresados de la ANUIES ${ }^{[1]}$. Algunos de estos estudios se complementan con otro tipo de técnica o instrumento como lo hicieron Burgos, Rodríguez y López (2011), Rodríguez y Valenzuela (2017), en Ecuador utilizaron variables socioeconómicas, impacto del empleo, pertinencia, relación de las personas graduadas y la facultad y, contribución de la educación recibida. Por su parte, Artigas y Moreiro (2003) consideran características demográficas, académicas, laborales, sociales, de servicios y de empleo, el grado de adecuación de la formación universitaria al mundo laboral y profesional, además de brindar información acerca de las posibles opciones a las cuales se enfrentarán los graduados en el área estudiada por ellos. Maya y Herrera (2012) dieron cuenta del estado del arte sobre los estudios de desempeño de graduados del nivel superior, encontrando que se utilizan para rediseñar currículos, estrategias didácticas

y emprender acciones de mercadeo. De aquí que, para desarrollar estos estudios existen diferencias por lo general en la metodología y en específico en las variables abordadas.

Además, se considera que los estudios de personas egresadas también permiten dar cuenta de la pertinencia de las Instituciones de Educación Superior (IES), así como permiten obtener información indispensable para acreditar o certificar planes y programas de estudio, procesos a los cuales se someten de manera constante las IES, sobre todo las públicas. Por tanto, son ejercicios que se deben ejecutar sistemáticamente. De aquí que el 
presente artículo tiene como objetivo analizar tres dimensiones de una encuesta de egresados que se aplica en la UMSNH que dan cuenta respecto al tránsito de la universidad al MT.

\section{MARCo TEÓRICO}

Los estudios de egresados están diseñados para recabar información sobre la percepción de los egresados respecto de su formación académica, así como de las posibilidades de empleo que tienen cuando tratan de insertarse en el MT. Medir la percepción de los egresados se considera el doble de importante pues, por una parte, evalúan la formación que recibieron y por otra son fuente de evaluación, dado los resultados laborales y profesionales que presentan al egresar.

Para Cuevas (2007) son considerados una alternativa para el autoconocimiento, la planeación y la consolidación de los programas y las instituciones educativas, así como evaluar y retroalimentar el currículo, para Contreras, Chávez y Víquez (2015), estos estudios permiten además: a) mejorar la planificación de las carreras ofertadas considerando el contexto en el que se desenvuelven los egresados; b) evaluar las aportaciones de las IES al desarrollo, es decir en su contexto inmediato a través de la acción de sus egresados; c) evaluar el grado de aceptación en el sector productivo que tienen los estudiantes al egresar; d) conocer el nivel de empleo, subempleo y desempleo de los egresados; y por último e) conocer la pertinencia y la vigencia de los planes y programas de estudio y, para Fresán (2003) "son una herramienta básica para la definición de políticas en el nivel regional, estatal e incluso nacional” (p. 23).

Para el caso de México, el modelo propuesto por la ANUIES es importante no solo porque en él se basan la mayoría de las IES a nivel nacional tanto públicas como privadas para recabar esos datos, lo es también porque obligatoriamente cualquier universidad que se quiere certificar, acreditar, evaluar o proponer un nuevo programa educativo (PE) tiene que contar con estos estudios. La encuesta que se aplica en la UMSNH, también se basa en dicho modelo, y se agregan tres dimensiones más: Responsabilidad Social Universitaria, clima universitario y recomendaciones para mejorar el perfil profesional.

Estos estudios, suelen ser complementados con estudios de MT para obtener información sobre la percepción de los egresados respecto a su formación, la organización académica y los planes de estudio, así como sobre los estudios de opinión de especialistas y empleadores sobre requerimientos actuales y futuros de los perfiles de formación. En los estudios de MT, se recaba información por medio de entrevistas, encuestas o implementación de grupos focales con empleadores, pues ellos representan al sector productivo quienes, por una parte, conocen sobre las características de la demanda laboral de las empresas y por otra, la valoración que los contratistas hacen sobre el desempeño de los egresados, información que permite detallar un panorama más certero sobre cómo está en ese momento histórico configurado el MT. De aquí que también sean utilizados para dar cuenta tanto de la pertinencia de un PE o de la pertinencia de una IES (Almonacid, Montes y Vásquez, 2009; Osorio, Martínez y Contreras, 2010; Valdés, Morales, Díaz, Sánchez y Cuallar, 2009).

En este sentido, la expansión experimentada por las IES en las últimas décadas, ha impactado de manera profunda en los MT. Machin y McNally (2007) consideran que ha cambiado la forma en que los empleadores contratan mano de obra altamente calificada y agregan que esta expansión se ha basado en la suposición de que la educación es buena para los individuos, no solo en términos económicos, sino para la sociedad en su conjunto, pues el MT es referido al mercado donde confluyen la oferta y la demanda de profesionistas, que se da en un momento y lugar determinado (ANUIES, 2003). Además, este tipo de mercado se considera parte de una estructura económica y que conlleva otras aristas como la intervención del gobierno, las condiciones laborales y salariales de los trabajadores, la vocación productiva de la región, la inversión en infraestructura, demografía, entre otros, por lo cual se considera un mercado complejo. En este sentido, para Planas (2013, citado por Ibarrola, 2014), el MT y el de la educación son dos tipos de mercados muy diferentes entre sí y que se ha pensado son reflejo uno del otro, sin embargo, responden a lógicas de interacción muy diferentes, 
al respecto, Ibarrola (2007) considera que sería utópico pensar en alinear las IES y el MT, sin embargo, aún hay mucho por hacer al respecto.

Otra característica atribuida a estos estudios consiste en el poder obtener información respecto a cómo es la vinculación entre las IES y los sectores productivos (Hernández y Rodríguez, 2015) ya que actualmente, se busca compaginar objetivos en las agendas de las IES y el sector productivo, en pro de mejorar algunas condiciones del MT e impulsar la ciencia, tecnología e innovación en la localidad así como en la inserción efectiva en la vida cultural y comunitaria en la que están instaladas (Rodríguez, 1999). En este sentido, se reconoce el peso conferido a las IES como promotoras del desarrollo al menos en el contexto inmediato y de la importancia de estos estudios para orientar políticas de desarrollo local o regional, es decir, es remitirse tanto a la responsabilidad social, así como a la pertinencia educativa, económica, política y social de las IES.

Ahora para Scheele y Brunner (2009), los graduados se consideran empleados de exitosa inserción en el MT una vez que reportan que trabajan ya sea a tiempo parcial, completo, o temporal; por su parte Chavoya y Reynaga (2011) argumentan que es el MT el que marca la pauta en cuanto al tipo de profesionista que se necesita y agregan, que cuando un MT funciona mal se afecta de forma negativa el crecimiento económico y el empleo de un país, siendo algunos de los costes económicos el desempleo o subempleo y el coste social derivado de ello. De aquí que la inserción laboral es un indicador de gran valor en estudios de este tipo, al considerar que quien cuenta con educación superior tiene mayor probabilidad de encontrar empleo y además de obtener mejores ingresos. Sin embargo, para Planas (2013) habría que considerar otros aspectos, como el perfil profesional y la estructura del sector productivo de cada entidad.

Si bien el panorama es complejo, expertos en el tema como Ibarrola (2014), proponen cuatro dimensiones de análisis para investigar las relaciones en distintos espacios laborales y diferentes instituciones escolares: a) la profundización de la estructura laboral de México como heterogénea, desigual, combinada; b) las políticas impulsadas por el gobierno federal sobre la formación escolar para el trabajo y la influencia de diferentes actores sociales en ellas; c) la manera como las instituciones escolares procesan la formación para el trabajo; y d) la transición del estudio al trabajo y las trayectorias escolares y laborales de jóvenes. En los dos últimos puntos es dónde se sitúan los resultados posteriormente presentados.

\section{Metodología}

El método utilizado es el cuantitativo, el tipo de diseño es no experimental de corte transeccional y de alcance descriptivo, pues este tipo de estudios permiten describir fenómenos, situaciones y contextos, así como presentar los resultados, pero sobre todo analizarlos ya sea de manera independiente o haciendo agrupaciones, logrando analizar de manera más exhaustiva los resultados obtenidos de la encuesta (Hernández Fernández y Baptista, 2010).

La muestra la constituyen egresados de la UMSNH, el muestreo fue no probabilístico intencional. El instrumento fue la encuesta de egreso que se aplica a nivel institucional. Es una escala tipo Likert, compuesta de doce variables y para los fines de este trabajo, se analizan solo tres: trayectoria y ubicación en el MT actual, empleo actual y satisfacción laboral, variables que se relacionan directamente con la aceptación de los egresados en el MT.

Se solicitó la base al departamento correspondiente, pero se entregó incompleta, ello pudo deberse a las leyes de protección de datos de identidad vigentes, sin embargo, se considera una limitante de este estudio. Se procedió a limpiarla y analizarla y se realizaron los análisis estadísticos en el programa SPSS versión 18 para Windows.

Respecto a la base de datos, esta constó de 11.444 registros del nivel superior, durante el periodo comprendido de 1972 hasta enero de 2017, mas en el periodo 2010 a 2017 fue donde se concentró el 74,5\% de la muestra. E1 54,19\% de los participantes son mujeres y un 45,81\% son hombres. Para el análisis de resultados los datos se agruparon conforme a la Clasificación Mexicana de Programas de Estudio (CMPE) ${ }^{[2]}$, ello con 
la finalidad de alinear los resultados bajo criterios nacionales que luego permitan hacer comparaciones por campo formativo. Bajo esta lógica, un 38\% de los encuestados pertenecen al campo de las Ciencias Sociales, Administrativas y Derecho, un 35\% al área de Salud, que de manera conjunta agrupan el $73 \%$ de los egresados encuestados (ver Figura 1).

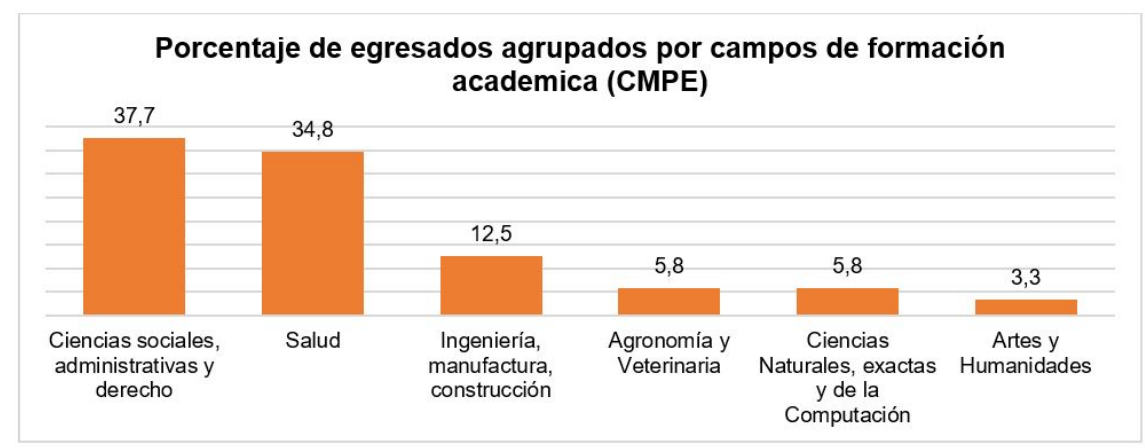

FIGURA 1

Agrupación por campo de formación académica

Fuente: Elaboración propia.

\section{Resultados}

\section{Trayectoria y ubicación en el mercado de trabajo}

Para Valenti y Varela (2003), las preguntas sobre cómo se incorporan los egresados al MT, sirven para conocer los tiempos insumidos en la búsqueda de empleo, los medios a través de los cuales se obtuvo el empleo y los factores que contribuyeron a la consecución de este. El 37,8\% encontró trabajo durante los primeros seis meses, un $37,5 \%$ reporta no haber encontrado empleo al momento de la entrevista, un $16,7 \%$ tardaron de seis meses a un año y un $8 \%$ tardo más de un año en obtener uno (ver Figura 2). Es decir que, poco más de la mitad de los que egresan, encuentran trabajo durante el primer año de egreso, que Valenti y Varela (1997) denominan como un segundo momento de inserción acelerada ${ }^{[3]}$. Por campo formativo, se puede apreciar que el porcentaje de inserción es menor en los campos de Artes y Humanidades y, Ciencias Naturales, Exactas y de la Computación.

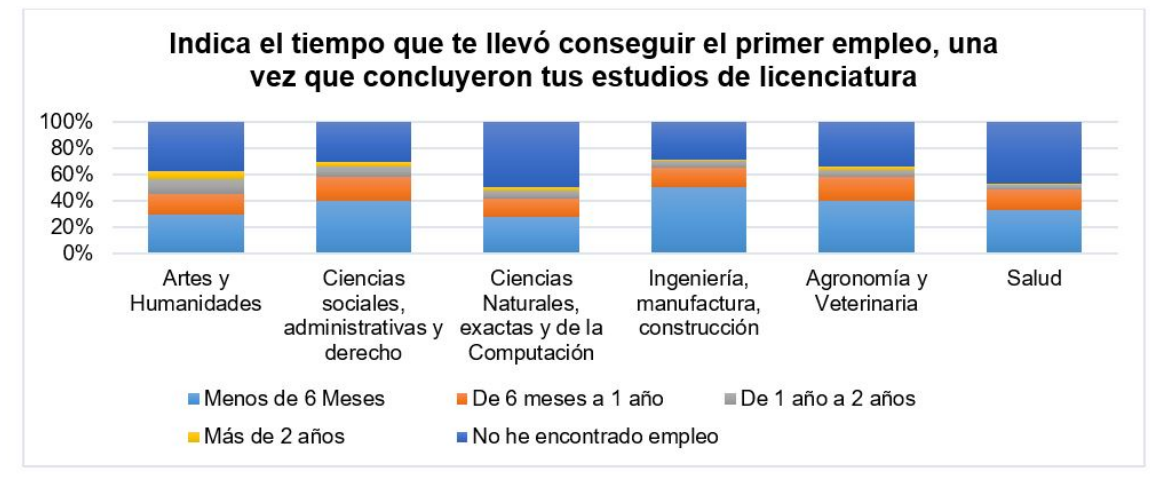

FIGURA 2

Tiempo que les toma conseguir empleo una vez que concluyen sus estudios de licenciatura Fuente: Elaboración propia 
Respecto a la pregunta ¿a qué le atribuyen la demora y/o dificultad para conseguir empleo? un 30,2\% lo atribuyó a no estar titulado, un $18,4 \%$ a otra circunstancia y un $18,1 \%$ a que estudiaron una licenciatura poco conocida, entre estos tres reactivos agrupan el $67,7 \%$ de las respuestas. Es de llamar la atención que el porcentaje más alto fue la opción no estar titulado, factor que afecta en los índices de calidad de las universidades, pues es un indicador de peso al momento de las acreditaciones. Ahora, cuando se les pregunta sobre el grado de relación entre sus estudios y el trabajo que ejecutaron en su primer empleo, este se evalúa positivamente.

La coincidencia entre formación y empleo se suele explicar desde la postura de que ciertos programas educativos tienen ventajas respecto a otras áreas (Ávila y Aguirre, 2005), además de que a través de esos datos se pueden conocer aspectos sobre cómo está configurado el MT en la región. En porcentajes entre un 35\% y un $62 \%$ percibió como total coincidencia siendo los más satisfechos los del área de ciencias de la salud. En el caso de las ciencias naturales, exactas y de la computación y, artes y humanidades fueron los que reportaron menor coincidencia entre sus estudios y su primer trabajo (ver Figura 3).

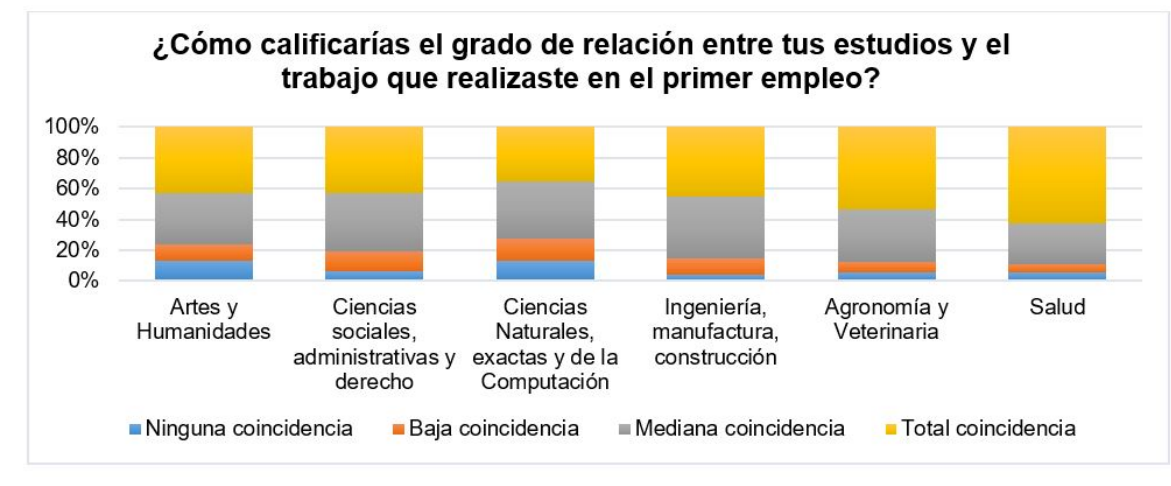

FIGURA 3

Percepción sobre el grado de relación entre sus estudios y el primer empleo

Fuente: Elaboración propia

Respecto al requisito de mayor peso para conseguir el primer empleo, en los seis campos fue el tener una licenciatura, seguido de tener experiencia previa. Opciones de respuesta con menores porcentajes fueron: aprobar el examen de selección, recomendación y por último, desempeño en la entrevista (ver Figura 4). El promedio por campo formativo, en el cual mencionan que contar con la licenciatura les permitió encontrar empleo fue de 36,9\%, quienes consideran que fue por aprobar examen de selección 24,4\%, a diferencia de resultados encontrados en la UAM por Valenti y Varela (1997), los cuales fueron de 44,8\% y un 36,8\%; respectivamente. Si bien las otras opciones de respuesta fueron elegidas en porcentajes menores, habría que tenerlas presentes. Por ejemplo, se ha encontrado que la experiencia profesional previa, es uno de los determinantes de la entrada al MT (Ruesga, da Silva y Monsueto, 2014) y los egresados de la UMSNH, en promedio el $24 \%$, seleccionó esta opción. 


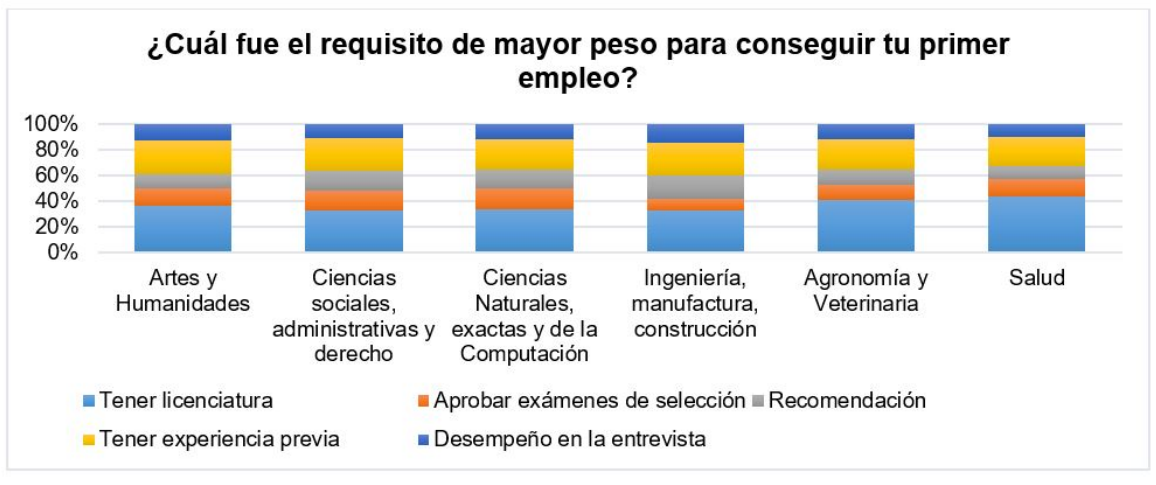

FIGURA 4

Gráfica sobre el requisito de mayor peso para conseguir empleo

Fuente: Elaboración propia

Además, los requisitos que consideran influyen en la obtención de un empleo, se encontró que el prestigio facilitó (41,4\%); la aceptación de la carrera en el MT facilitó (69,0\%); la coincidencia de la carrera con las necesidades de la empresa o institución para un 80,1\% también facilitó. La experiencia previa, la edad, el género y las responsabilidades familiares influyeron en menor medida.

Ahora, de la muestra, un $44,5 \%$ reportó no tener trabajo y un 50,2\% sí contaban con empleo al momento de contestar la encuesta. Al contrastar estos datos con el campo formativo, sin perder de vista que el área de Ciencias Sociales y de Salud concentran la mayor parte de los encuestados, se puede observar que existen diferencias en cuanto a los porcentajes de empleo y desempleo por área. Las áreas que concentran más egresados con empleo, son las Ciencias Sociales, Administrativas y de Derecho; Ingeniería, Manufactura y Construcción; Agronomía y Veterinaria. Los campos en los cuales se reportó más desempleo durante el primer año de egreso fueron el área de la Salud y las Ciencias Naturales, Exactas y de la Computación, ambas con mayor porcentaje de egresados que no reportaron estar empleados en comparación con los que sí lo estaban (ver Figura 5).

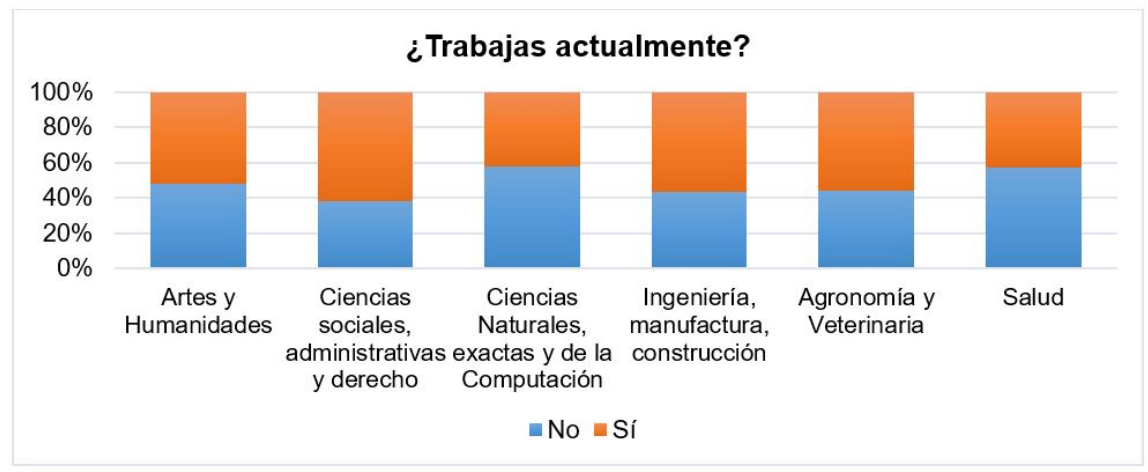

FIGURA 5

Gráfica del estatus de empleo al momento de contestar la encuesta Fuente: Elaboración propia

\section{Empleo laboral actual}

Cuando se preguntó si trabajan en algo distinto a su primer empleo, el 63\% reporta que mantiene su mismo trabajo y el $37 \%$ cuenta con un trabajo diferente. Respecto a si su puesto de trabajo mejoró una vez que egresaron de la licenciatura se encontró que para el 56\% mejoró, para el 21,5\% está igual y para un 2,1\% 
empeoró. Lo cual lleva a considerar que, contar con una licenciatura facilita el acceder a un trabajo y Valenti y Varela, (1997) consideran que es un factor de movilidad social.

En relación con si el nivel de ingresos mejoró, en comparación entre su primer trabajo una vez que egresaron y el empleo actual, para un 55,6\% mejoró, para el 22,5\% está igual, para un 3,5\% empeoró y un 18,5\% eligió la opción de no aplica. Por campo formativo mejoró el ingreso principalmente para los de Ingeniería, Manufactura y Construcción, para los de Agronomía y Veterinaria y, para las Ciencias Sociales, Administrativas y de Derecho (ver Figura 6).

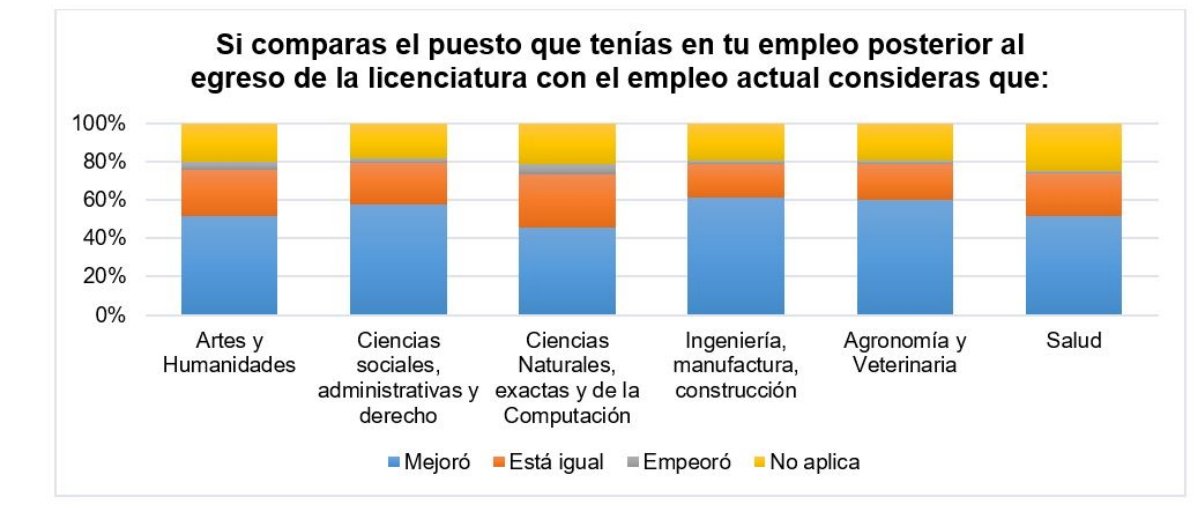

\section{FIGURA 6 \\ Gráfica de comparación entre el puesto adquirido al egresar y el empleo actual \\ Fuente: Elaboración propia}

Estos resultados sugieren la existencia de áreas de formación que son mejor remuneradas una vez que egresan. Si estos datos se desglosaran por programa educativo se podría indagar con más detalle a qué se debe que en ciertas áreas no están adquiriendo mejores ingresos y que muy probablemente engrosen las filas del subempleo o en otro mejor panorama, se estén auto empleando en sus áreas de formación.

Ahora, al 53,8\% que reportó tener un empleo se les preguntó sobre su posición en la ocupación. Un 84,9\% reportó ser empleado; un $6,42 \%$ propietario y un $8,65 \%$ ser trabajador independiente. ${ }^{[4]}$ Respecto al tamaño de empresa donde laboran, el $42 \%$ trabaja en alguna microempresa, el $20 \%$ en pequeñas empresas, el $10 \%$ en medianas empresas y el $28 \%$ en empresas grandes, es decir que, poco más del $60 \%$ de los egresados se emplean en micro y pequeñas empresas (ver Figura 7). Datos que concuerdan con los presentados por el Instituto de Información Estadística y Geográfica [INEGI] (2018) para el estado de Michoacán, respecto a que más del $90 \%$ de las empresas registradas, son micro y pequeñas empresas. 


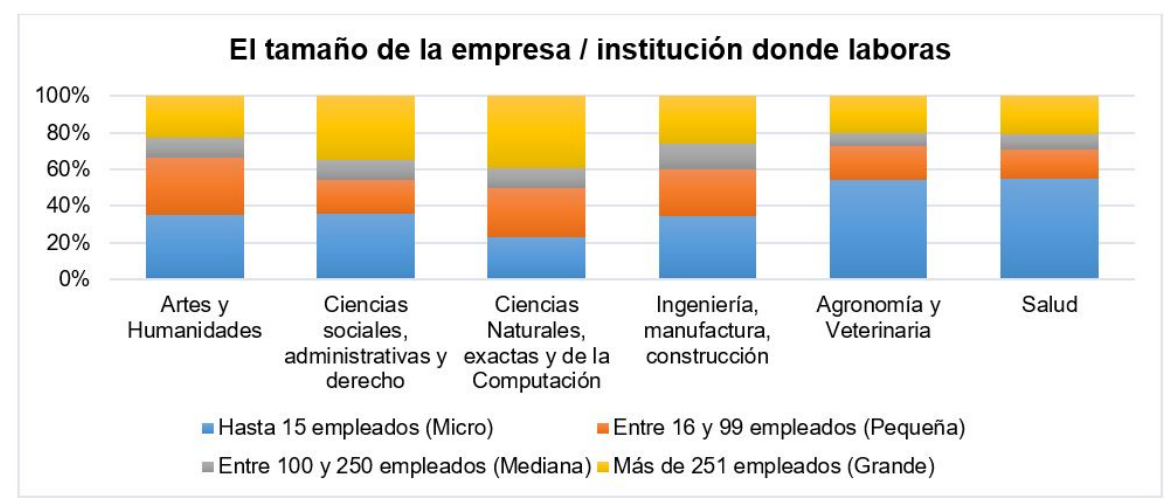

\section{FIGURA 7 \\ Gráfico de datos sobre el tamaño de la empresa \\ Fuente: Elaboración propia}

Si los datos se revisan por campo formativo se encuentra que los campos de Agronomía y Veterinaria, y Salud son los que mayores porcentajes de empleo reportan en micro empresas, a diferencia del campo de Ciencias Naturales y Exactas que reportan mayores porcentajes de empleo en empresas grandes. Si la variable tamaño de la empresa se contrasta con la posición que ocupan en su trabajo, se puede observar que en las microempresas es dónde se encuentran más trabajadores independientes y propietarios, a diferencia de los otros tipos de empresas (ver Figura 8).

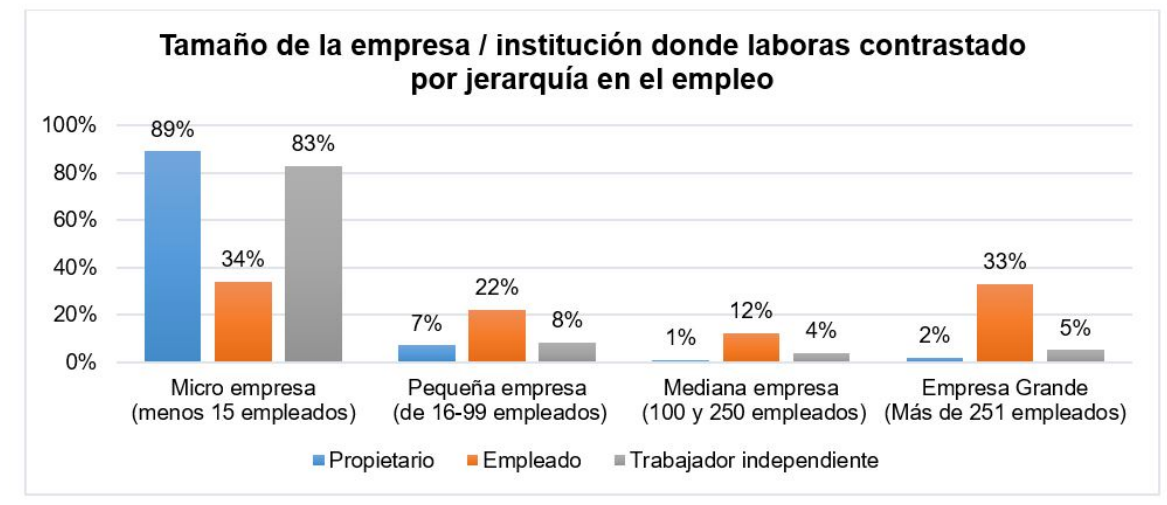

FIGURA 8

Gráfico que contrasta el tamaño de la empresa en la que laboraban con la posición en su empleo Fuente: Elaboración propia.

En este sentido, se considera que las micro empresas están empleando y, a su vez están siendo creadas por los egresados. Lo que no se conoce con la encuesta aplicada es si las micro empresas son creadas antes o después de egresar. Ahora respecto a la coincidencia entre los estudios y su actividad laboral un $58 \%$ consideró que existe una total coincidencia, un $25,15 \%$ mediana coincidencia, un $8,50 \%$ baja coincidencia y un 7,62\% ninguna coincidencia. Los campos formativos de la salud; agronomía y veterinaria; e ingeniería, manufactura y construcción, reportaron mayores porcentajes de total coincidencia (ver Figura 9).

Si se contrastan los datos del sector económico por campo formativo, se encontraron egresados que laboran en un trabajo afín a su área, pero el área que reportó mayor coincidencia son los del campo de la salud. Estos datos coinciden con los emitidos por el Observatorio Laboral [OLA] en el año 2014, para el estado de Michoacán, en donde se reportan porcentajes arriba del 75\% de los empleados en ese campo de la salud, laborando en su área y en porcentajes menores a 18\% de profesionistas con este perfil, están subempleados. 


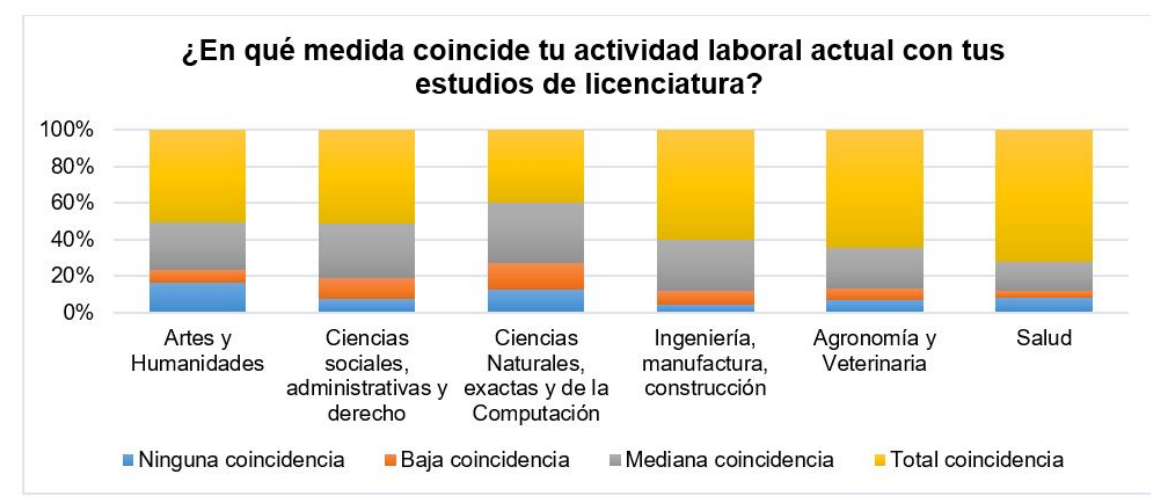

FIGURA 9

\section{Gráfica de coincidencia entre actividad laboral y estudios de licenciatura}

Fuente: Elaboración propia

Respecto al tipo de contrato laboral, un 22,6\% reportó tener un contrato indeterminado, un 13,5\% un contrato temporal, un $8,6 \%$ no contaba con contrato y un 5,7\% reportó ser propietario independiente, por obra determinada o tiene otro tipo de contrato. Cabe recordar que un $49,5 \%$ que no contestaron estos ítems fue por no contar con un trabajo al momento de ser encuestados. Respecto al sector económico un $13,1 \%$ reportó laborar en los servicios de salud, un $8 \%$ en los servicios de gobierno, un 7,6\% en los servicios profesionales o técnicos y un $6,6 \%$ en servicios educativos. Con menores porcentajes se eligieron otras opciones (ver Figura 10).

Si se contrastan los datos del sector económico por campo formativo, se encontraron egresados que laboran en un trabajo afín a su área, pero el área que reportó mayor coincidencia son los del campo de la salud. Estos datos coinciden con los emitidos por OLA para el estado de Michoacán en el año 2014, en donde se reportan porcentajes arriba del 75\% de los empleados en ese campo de la salud, laborando en su área y en porcentajes menores a $18 \%$ de profesionistas con este perfil, están subempleados. Los del campo formativo de Artes y Humanidades, en su mayoría laboran en el ramo de la educación (69\%) y en el sector comercio (10\%).

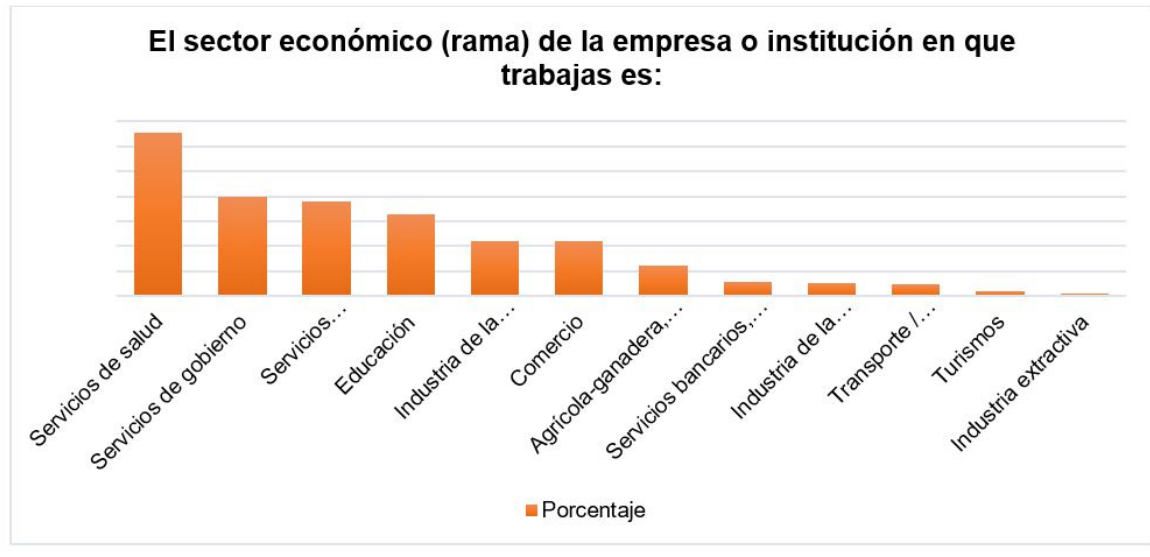

FIGURA 10

Gráfica del sector económico o rama de la institución en la que laboraban, al momento de contestar la encuesta Fuente: Elaboración propia

Los resultados a la pregunta sobre el medio a través del cual encontraron empleo en las seis áreas en mayor porcentaje mencionaron que fue por recomendación y solo en las áreas de Agronomía y Veterinaria y Salud, el porcentaje mayor se concentró en búsqueda de empleo a través de entrega de currículum de manera directa 
(ver Figura 11). El papel que tienen las bolsas de trabajo publicadas por la institución cabría esperar que fuera más dinámico, sin embargo no en todas las facultades de la universidad cuentan con este servicio.

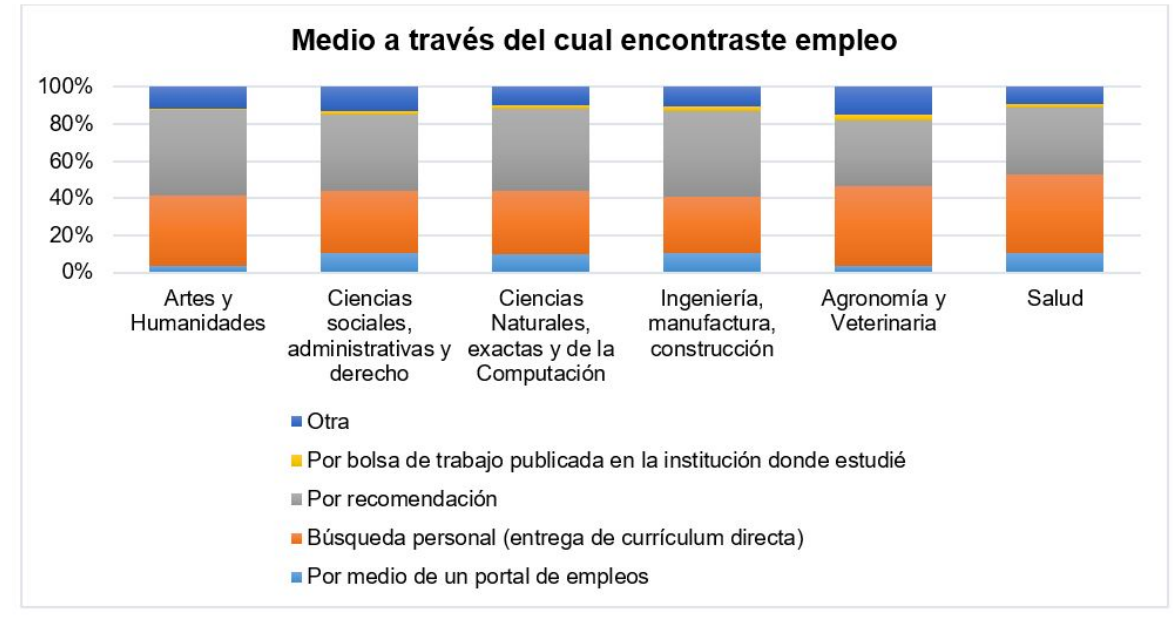

FIGURA 11

\section{Medio por el cual obtuvieron empleo contrastado por campo formativo}

Fuente: Elaboración propia

Ahora bien, cuando se les preguntó a quienes reportaron no tener empleo, cuál consideraban era la razón principal para no contar con uno, se encontró que: un 21,6\% eligió la opción de no he sido seleccionado dentro de los procesos de contratación, un 10,7\% no consiguió empleo porque se dedicó a seguir estudiando, un $8,9 \%$ no había buscado, un 7,6\% eligió la opción otra y en menores porcentajes por dedicarse al hogar o porque no lo necesita. Este dato de estudiantes que decidieron seguir estudiando equivale al $10 \%$ del total de la muestra encuestada. Por campo formativo, los de las áreas de Ciencias Naturales, Exactas y de la Computación eligieron en mayor proporción el seguir estudiando (42,2\%) seguido del campo de Artes y Humanidades (30,5\%). El 52\% del campo de Ciencias Sociales, Administrativas y Derecho eligió la respuesta de no haber sido seleccionado en los procesos de contratación (ver Figura 12).

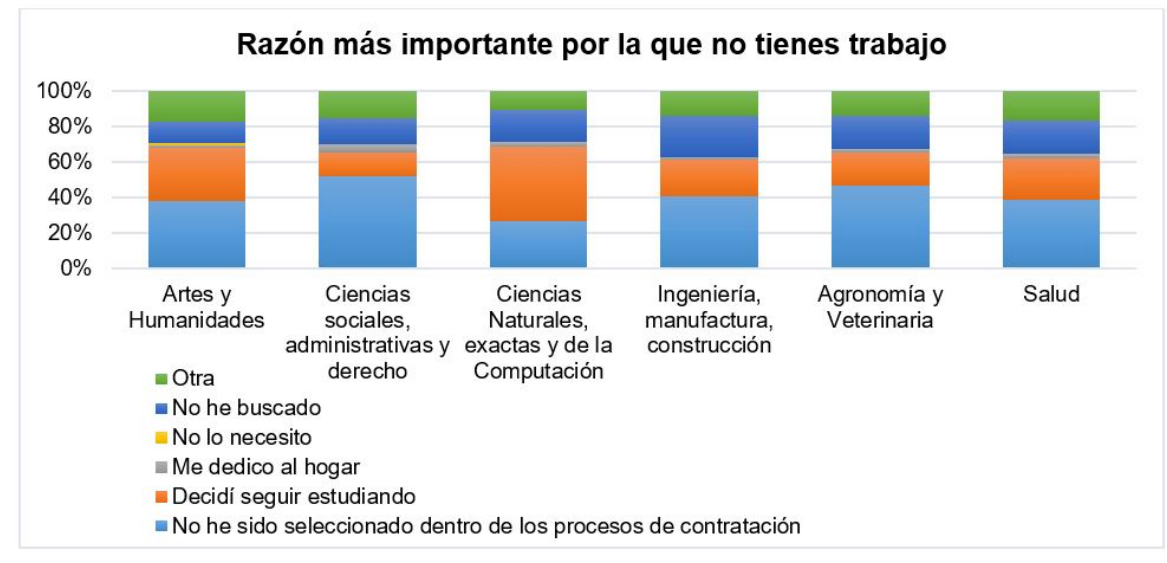

FIGURA 12

Gráfica motivos por los cuales consideran que no tienen trabajo

Fuente: Elaboración propia

Los resultados hasta aquí mostrados caracterizan la trayectoria que efectúan los estudiantes una vez que egresan y el proceso de consecución de un empleo y para complementar estos resultados se describe la satisfacción laboral, aspecto que ha sido relacionado con programas bien organizados, con buenos contenidos de enseñanza y con énfasis en el trabajo práctico (Gil, García y Santos, 2009). 


\section{SATISFACCIÓN LABORAL}

Se parte de una pregunta general, ¿qué tan satisfecho estás con los siguientes aspectos de tu trabajo? Posteriormente se les presenta un listado con doce oraciones en las cuales responden eligiendo entre cuatro opciones: nada satisfecho, poco satisfecho, muy satisfecho y totalmente satisfecho. Los ítems responden a las dos perspectivas utilizadas para abordar las investigaciones referentes a la satisfacción laboral: la satisfacción como un estado emocional, un sentimiento, una actitud o una respuesta afectiva hacia el trabajo y la visión de los que la consideran como el resultado de una comparación o ajuste entre expectativas, necesidades o resultados actuales del puesto y las prestaciones que el trabajo les ofrece en realidad (Sánchez, Artacho, Fuentes y López, 2007). Además de estar basadas en el documento de la ANUIES (2003).

Para presentar los resultados se agruparon los doce indicadores, separando las respuestas por campo formativo. En cinco de los seis campos formativos, en mayor porcentaje, manifestaron una total satisfacción, la opción muy satisfecho fue la segunda respuesta más elegida (también en cinco de los seis campos formativos). Y en proporciones menores al $10 \%$ se encontró la respuesta de poco satisfecho. Respecto a la opción de nada satisfecho, fue una opción muy poco seleccionada, pues solo en el área de artes y humanidades agrupó al 1\% en esa opción. Los que manifiestan mayor satisfacción laboral son los de los campos: agronomía y veterinaria 59\%, seguidos del área de la salud 55\% y del área de Ingeniería, manufactura y construcción 52\%. Y los menos satisfechos respecto a su trabajo, se puede decir, son los del campo formativo artes y humanidades, y este fue el único campo formativo en el cual su mayor porcentaje se concentró en la respuesta de muy satisfecho (ver Figura13).

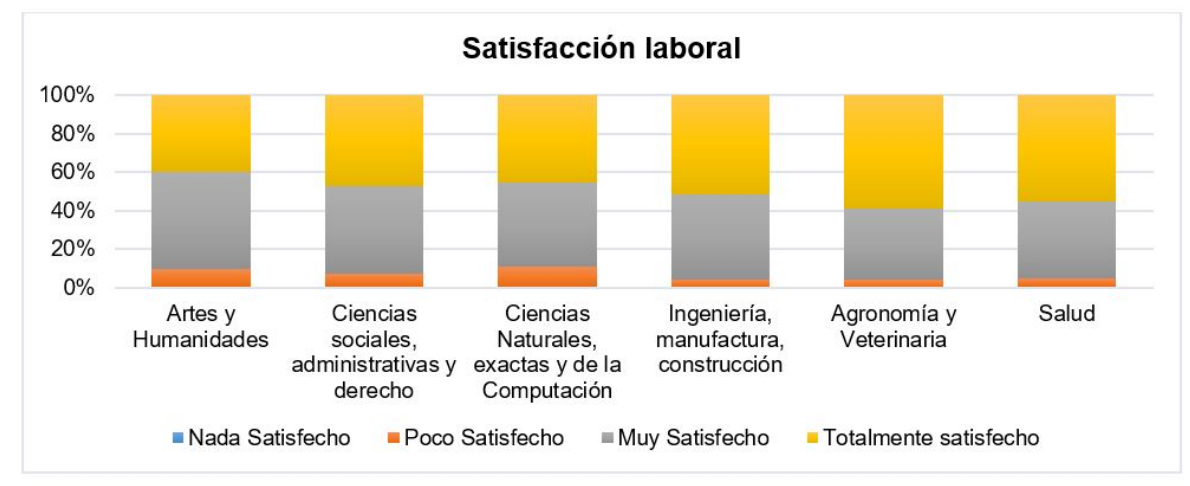

FIGURA 13

Gráfica de agrupación de los doce indicadores que miden la variable satisfacción laboral Fuente: elaboración propia

De manera específica se describirán solo dos ítems, el referido al salario y el alusivo a la posición jerárquica alcanzada, por ser ítems que tuvieron más variabilidad en los datos. Respecto al salario, los porcentajes más altos de respuesta se concentraron en la opción poco satisfechos, lo que no sucedió con los otros ítems que evalúan la percepción de la satisfacción, siendo los de artes y humanidades los que remiten menos satisfacción respecto al salario (ver Figura 14). 


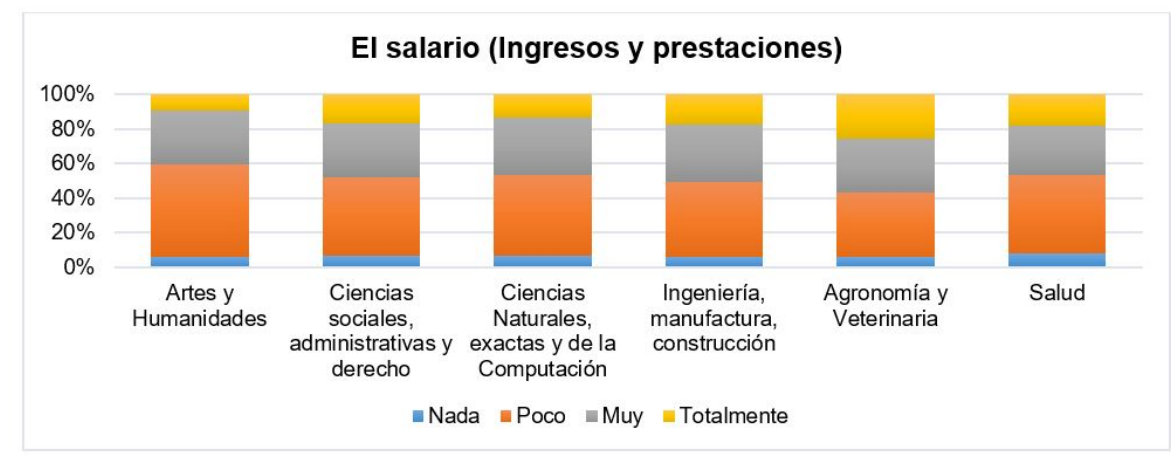

FIGURA 14

Percepción sobre la satisfacción respecto al salario por campo formativo Fuente: elaboración propia

En el caso de la satisfacción respecto a la posición jerárquica alcanzada, la opción más elegida fue la de estar poco satisfechos; siendo de nuevo los del campo de Artes y Humanidades los que reportaron mayor porcentaje de insatisfacción (43,4\%), seguido del campo de ciencias naturales, exactas y de la computación (40,8\%) (ver Figura 15 ).

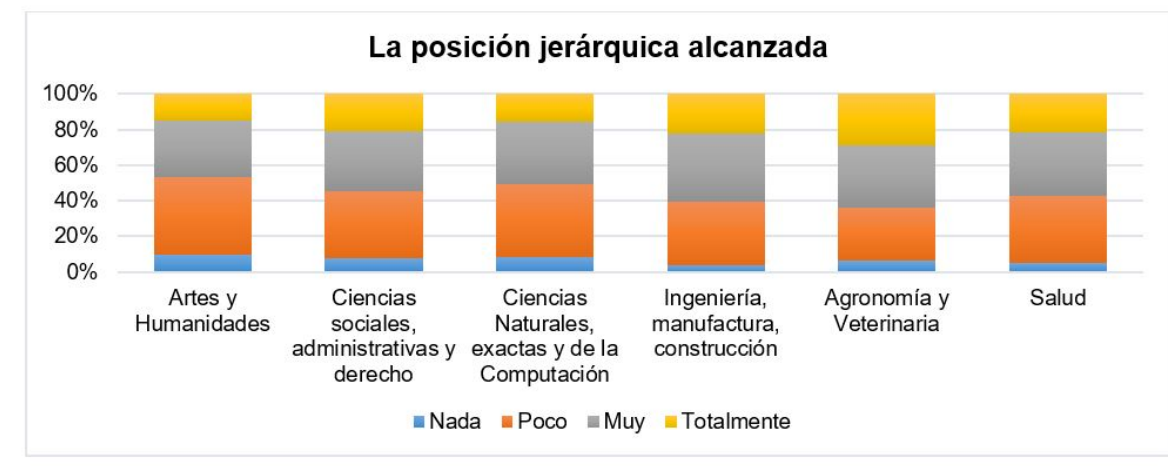

FIGURA 15

Satisfacción respecto a la posición jerárquica alcanzada

Fuente: elaboración propia

\section{DISCUSIÓN Y CONCLUSIONES}

El objetivo de esta investigación consistió en analizar tres dimensiones de la encuesta de personas egresadas que se aplica en la UMSNH que dan cuenta respecto al tránsito por el cual pasa el estudiantado una vez que egresa de la universidad al MT. Tarea nada fácil, desde la concientización de la población estudiantil y del profesorado sobre la importancia de estos estudios, hasta la aplicación y uso de la base de datos. Conocer esta información, permite identificar aspectos para mejorar los planes y programas de estudios, así como apoyo en implementar políticas públicas que faculten mejorar las condiciones del MT para los futuros profesionistas, sin embargo, una dificultad para analizar la información es que, en los estudios desarrollados por otros investigadores, se utilizan diferentes variables y metodologías. Aspecto que hace que sea casi imposible comparar los resultados.

En este sentido, si bien no pueden ser del todo comparables, los resultados permiten tener un panorama del tránsito de la universidad al MT, así como algunas características sobre cómo se conforma el MT en el estado. La UMSNH en el estado, en la actualidad capta mayor porcentaje de estudiantes, no solo aspirantes del propio estado, sino de estados vecinos, reportando una matrícula de 39.390 para el ciclo escolar 2016-2017 en 
los niveles de licenciatura y posgrado (ANUIES, 2017). Al ser una universidad de gran renombre en la región, en los resultados se encontró que aproximadamente la mitad de quienes egresan no encuentran trabajo, al menos no en el primer año. Al respecto, Machin y McNally (2007), consideran que no se puede decir que exista exceso de personas egresadas y que por ello sea más difícil colocarse en algún empleo, más bien implicaría centrar la mirada en la estructura productiva de cada región.

Ahumada y Oregón (2017), reportan que el $76 \%$ consigue emplearse en los primeros seis meses; Valenti y Varela (1997) reportan una inserción de 59,6\% también para los primeros seis meses, y al año un 80,1\% del estudiantado de la Universidad Autónoma Metropolitana (UAM) habían encontrado un trabajo, lo cual sugiere que los egresados de la Michoacana están tardando o se les dificulta obtener un empleo. Otro aspecto, es que algunas carreras reportan una entrada acelerada al MT, como el campo de las ingenierías en el país (Valenti y Varela, 1997; OLA, 2014), aspecto que ocurre también para los campos de ciencias sociales, administrativas y de derecho y, en agronomía y veterinaria de la UMSNH. La inserción laboral, es uno de los indicadores de mayor importancia en estos estudios y se puede decir que es un foco rojo por atender, pues este tipo de problemas se considera, generan dudas sobre la eficiencia de la inversión en educación y captación y por tanto sobre las perspectivas del crecimiento económico (Weller, 2006).

Sobre los requisitos que les permitieron encontrar empleo, el de mayor peso fue, el contar con una licenciatura, seguido de la experiencia previa. Tener una licenciatura para acceder a salarios mejor remunerados se ha considerado como una visión credencialista lo cual ha llevado a que se cuestione la calidad de la educación, pese a esta postura, se ha encontrado que la educación del nivel terciario es un factor dinámico de movilidad social y de ascenso ocupacional de los egresados (Muñoz, 1996; Valenti y Varela, 1997). La experiencia previa, es factor importante para emplearse, resultados que concuerdan con los de Ruesga et al. (2014) quienes encontraron que es un determinante para encontrar empleo.

En este mismo sentido, la coincidencia de la carrera con las necesidades de la empresa (o institución), así como la aceptación de la carrera en el MT también facilitó obtener un empleo, por ello se considera importante que exista congruencia entre la formación profesional y algunas características del mercado de trabajo. Al respecto se dice que un desfase entre las características de la educación y de la demanda laboral tiende a cerrar el canal de la movilidad social, con lo que se agravan los problemas estructurales de la mala distribución del ingreso en la región (Weller, 2006), de aquí que la UMSNH tienen un gran desafío ante lograr relacionarse y coordinarse con el MT sin perder autonomía, en pro de sus egresados. En este sentido, el que se estén empleando en micro o pequeñas empresas principalmente, lleva a confirmar que el MT en Michoacán está compuesto en su mayoría por micros y pequeñas empresas (Mypes) y que de acuerdo con el INEGI (2018) más del 90\% son de este tipo en el país y se acentúa un poco más en el estado de Michoacán. El hecho que los egresados sean o trabajadores independientes o propietarios, aun cuando se concentren en microempresas, puede sugerir que la educación superior contribuye en formar emprendedores y que no solo egresan para emplearse.

Por otra parte, es importante recuperar información sobre la coincidencia entre formación y empleo, sin embargo, se desconocen cuántos de estos egresados están subempleados, pues de acuerdo con datos del OLA (2014) para el estado de Michoacán, existen al tos porcentajes de subempleo, y son pocas las áreas que reportan porcentajes altos de coincidencia entre su formación y el empleo adquirido. La coincidencia entre formación y empleo Ávila y Aguirre (2005) la explican desde la postura de que ciertos programas educativos tienen ventaja respecto a otras áreas, por la factibilidad de poder trabajar por cuenta propia, así como de generar empleo. Los datos arrojados para el campo de salud, coinciden con los emitidos por Vries y Navarro (2011) quienes encontraron que a nivel Latinoamérica, más del 30\% de los egresados de este campo trabajan por cuenta propia y en el caso de México, es uno de los países que registró porcentajes más altos en comparación con otros países latinoamericanos. En el caso de campo formativo de ciencias naturales, exactas y de la computación el porcentaje de coincidencia es de 40\% en comparación del reportado por OLA (2014) a nivel estatal (52\%). 
La diferencia es que, a nivel estatal en este campo se reportan mayores porcentajes de coincidencia y en el caso particular de la UMSNH es el que menor coincidencia reporta.

Entonces, de acuerdo con la percepción de los egresados, los planes y programas educativos en los que se forman, los proveen de conocimientos, habilidades o destrezas para aplicarlos en el campo laboral, y se puede decir que, el problema central en el caso de México, no parece ser el acceso a la educación superior o la formación que las universidades ofrecen (Vries y Navarro, 2011), para ellas el problema está en la fuerte desigualdad social y económica dentro del mercado de profesionistas del país.

Los resultados encontrados sobre la satisfacción, pueden ser considerados como una fortaleza de la institución, pues esta se relaciona con los programas bien organizados, con buenos contenidos de enseñanza y con énfasis en el trabajo práctico (Mora, García y Vila, citados por Gil et al., 2009), además se considera que cuando los egresados están satisfechos en su empleo, ello lleva a pensar de manera subjetiva que los estudiantes tienden a estar satisfechos con los efectos que sus estudios han tenido sobre su ocupación actual (Planas, 2013). Respecto a la satisfacción con el trabajo, remitieron estar satisfechos en su empleo, sin embargo, hubo dos aspectos en los cuales estuvieron insatisfechos, el salario y la posición jerárquica alcanzada. Aspectos que no dependen directamente de la Universidad, sino de la estructura del MT, tanto a nivel local como nacional. En este mismo sentido, pese a que reportaron estar insatisfechos con el ingreso percibido, para un 55,6\% de los que reportaron tener un empleo, mejoró el ingreso al egresar. Al respecto Machin y McNally (2007), sostienen que en diversos estudios se muestra una tendencia a adquirir mejores puestos una vez que concluyen sus estudios y así mismo les permite acceder a un mejor ingreso.

En conclusión, la importancia de estos estudios, por una parte, es tener retroalimentación constante de la universidad y por otra, orientar o generar propuestas que permitan mejorar la vinculación y establecer líneas de trabajo entre las IES y los representantes de la estructura productiva que tienen injerencia directa en la conformación de los MT, como por ejemplo, diversificar el MT, así como desarrollar proyectos que promuevan el uso sustentable y responsable de la riqueza que tiene el estado de Michoacán, con la finalidad de influir de manera favorable en el desarrollo social al menos del contexto inmediato.

\section{REFERENCIAS}

Ahumada, F. N. y Obregón C. I. (noviembre, 2017). Seguimiento a egresados: un estudio diagnóstico en la Benemérita y Centenaria escuela normal del estado de San Luis Potosí, Congreso Nacional de Investigación Educativa COMIE. Trabajo presentado en el congreso llevado a cabo en San Luis Potosí, México. Recuperado de http://www.com ie.org.mx/congreso/memoriaelectronica/v14/doc/1227.pdf

Almonacid, P., Montes, I. C., y Vásquez, J. J. (2009). Un análisis factorial para evaluar la pertinencia de un programa académico desde la perspectiva de los graduados: un estudio de caso. Ecos de Economia, 13(29), 97-126.

Artigas, C., y Moreiro, J. (2003). Mercado de trabajo en Biblioteconomía y Documentación. Estudios sobre la inserción laboral de los titulados universitarios. El Profesional de la Información, 12(1), 4. Recuperado de http://eprints .rclis.org/13045/

Asociación Nacional de Universidades e Instituciones de Educación Superior [ANUIES] (2017). Instituciones de Educación Superior. México: ANUIES. Recuperado de http://www.anuies.mx/anuies/instituciones-de-educac ion-superior/

Asociación Nacional de Universidades e Instituciones de Educación Superior [ANUIES]. (2003). Esquema básicopara estudios de egresados. México: Serie investigaciones.

Asociación Nacional de Universidades e Instituciones de Educación Superior [ANUIES]. (2000). La educación superior en el siglo XXI: líneas estratégicas de desarrollo, una propuesta de la ANUIES. México: ANUIES. Recuperado de http://planeacion.uaemex.mx/InfBasCon/LaEducacionSuperiorenelSigloXXI.pdf

Ávila, M., y Aguirre, C. (2005). El seguimiento de los egresados como indicador de la calidad docente. REIFOP, 8(3), $1-5$. 
Burgos, B., Rodríguez, A. y López, K. (2011). Estudio de pertinencia de la oferta educativa de la Universidad de Sonora. México: Colección de investigación educativa.

Chavoya, M. L., y Reynaga, S. (2011). Las licenciaturas en Jalisco, México: crecimiento, diversificación y complejización de la educación superior y su impacto en la pertinencia. Espacio Abierto, 20(2), 289-311. Recuperado de http:/ /www.redalyc.org/articulo.oa?id=12218869004

Contreras, O., Chávez, M., y Víquez, J. (2015). Evaluación externa de la maestría en docencia para la educación media superior a través del seguimiento de sus egresados, XIII Congreso Nacional de Investigación Educativa. Trabajo presentado en el congreso llevado a cabo en Chihuahua, México. Recuperado de http://www.comie.org.mx/co ngreso/memoriaelectronica/v13/doc/1592.pdf

Cuevas, R. (2007). Estudio de seguimiento de egresados de la licenciatura en psicología educativa (Tesis de maestría). Universidad Pedagógica Nacional, México. Recuperada de http://200.23.113.51/pdf/24569.pdf

Fresán, M. (2003). Los estudios de egresados. Una estrategia para el autoconocimiento y la mejora de las IES. En ANUIES (Ed.), Esquema básico para estudios de egresados. Propuesta (pp.19-32). México: Serie investigaciones.

Gil, J., García, E., y Santos, C. (2009). Miradas retrospectivas de los egresados sobre la educación superior. Revista de Investigación Educativa, 27 (2), 371-393.

Hernández, J., y Rodríguez, J. (2015). La pertinencia de la educación desde la perspectiva de los estudiantes en una universidad pública mexicana. Cuadernos de Investigación Educativa, 6 (1), 33-51.

Hernández, R., Fernández, C., y Baptista, L. (2010). Metodología de la Investigación. México: Mc. Graw Hill.

Ibarrola, M. (2014). Repensando las relaciones entre la educación y el trabajo: Una reflexión basada en investigaciones realizadas en México. Ca. CEDES Campinas, 34 (94), 367-383. http://dx.doi.org/10.1590/S0101-32622014 000300006

Ibarrola, M. [UNEDAL productor]. (2007). Ibarrola segunda y última [Archivo de video]. Recuperado de https://w ww.youtube.com/watch?v=imd3aOJbAiY

Instituto de Información Estadística y Geográfica [INEGI]. (2018). Directorio de empresas y establecimientos. México: INEGI. Recuperado de https://bit.ly/2w1s4JF

Machin, S. y McNally, S. (2007). Tertiary education systems and the labour market. A paper commissioned by the Education and Training Policy Division (Tertiary Review). París: OECD. Recuperado de https://bit.ly/2LYn $\mathrm{U} 0 \mathrm{i}$

Maya, J. I., y Herrera, M. E. (2012). Aproximación al estado del arte sobre los estudios de desempeño de graduados de programas de educación superior. Revista virtual Universidad Católica del Norte, 36, 127-157. Recuperado de h ttps://www.researchgate.net/publication/237075758

Muñoz, C. (1996). Determinantes de la empleabilidad de los jóvenes universitarios y alternativas para promoverla. Revista Papeles de Población, 12(49), 75-89.

Observatorio Laboral [OLA]. (2014). Panorama anual. Resumen ejecutivo. 2013-2014. Recuperado de http://bit.ly $/ 2 \mathrm{H} 348 \mathrm{Mj}$

Osorio, E., Martínez, S. y Contreras, E. (2010). Estudio de pertinencia social del programa Educativo de Químico de la Universidad Autónoma del Estado de México. Educación química, 21 (1), 22-27.

Planas, J. (2013). Los itinerarios laborales de los universitarios y la calidad de su inserción profesional. Revista de la Educación Superior, 42(165), 31-62. Recuperado de https://www.redalyc.org/pdf/604/60428314003.pdf

Ranking Educativo QS. (04 de octubre de 2017). Las mejores universidades públicas de México. Universia. Recuperado de https://bit.ly/1hUMAx1

Rodríguez, K. L., y Valenzuela, M. G. (2017). Estudio de seguimiento a personas graduadas de postgrados de la Facultad de Ciencias Sociales y Humanísticas (FCSH) de la Escuela Superior Politécnica del Litoral (ESPOL). Revista Educación 41(1), 1-18. doi: http://dx.doi.org/10.15517/revedu.v41i1.17885

Rodríguez, R. (1999). La Educación Superior en el Siglo XXI. Lineas Estratégicas de Desarrollo. Una Propuesta de la ANUIES. México: ANUIES. Recuperado de http://publicaciones.anuies.mx/pdfs/revista/Revista113_S5A2E S.pdf 
Ruesga, S. M., da Silva, J., y Monsueto, S. E. (2014). Estudiantes universitarios, experiencia laboral y desempeño académico en España. Revista de Educación, 365, 67-95. http://doi.org/10.4438/1988-592X-RE-2014-365-265

Sánchez, S., Artacho, C., Fuentes, T., y López, T. (2007). Análisis de los determinantes Estructurales de la Satisfacción Laboral. Aplicación en el Sector Educativo. Estudios de Economía Aplicada, 25(3), 867-900.

Scheele, J., y Brunner, J.J. (2009). Educación terciaria y Mercado laboral: Formación profesional, empleo y empleabilidad. Revisión de la literatura internacional, Chile :Universidad Diego Portales, Centro de políticas Comparadas de Educación. Recuperado de https://bit.ly/2EnGb0L

Secretaría de Educación Pública. (2017). Principales cifras del sistema educativo nacional 2016-2017. México, D.F.: Secretaría de Educación Pública. Recuperado de http://planeacion.sep.gob.mx/estadisticaeindicadores.aspx

Valdés, A., Morales, I., Díaz, J., Sánchez, A., y Cuallar, R. (2009). Criterios de evaluación de la variable "pertinencia e impacto social" específicos para la carrera de Medicina. Educación Médica Superior, 23(3) Recuperado de htt p://bit.ly/2V1ZWQR.

Valenti, G., y Varela, G. (1997). El sistema de evaluación de las IES en México. Política y Cultura, (9), 131-147.

Valenti, G., y Varela, G. (2003). Construcción analítica del estudio de egresados. En ANUIES (Ed.), Esquema básico para estudios de egresados. Propuesta (pp.33-52). México: Serie investigaciones.

Vries, W., y Navarro, Y. (2011). ¿Profesionistas del futuro o futuros taxistas? Los egresados universitarios y el mercado laboral en México. Revista Iberoamericana de Educación Superior, 2 (4), 3-27. Recuperado de https://www.red alyc.org/articulo.oa?id=299124247001

Weller, J. (2006). Problemas de la inserción laboral de la población juvenil en América Latina. Papeles de Población, 12(49), 9-36. Recuperado de http://www.redalyc.org/articulo.oa?id=11204901

\section{Notas}

[1] La ANUIES es una asociación no gubernamental, que entre sus fines tiene el promover el mejoramiento integral de las tres funciones sustantivas de toda IES: docencia, investigación y extensión de la cultura y que respalda comités evaluadores y acreditadores de la educación superior en el país.

[2] El Instituto Nacional de Estadística y geografía [INEGI], clasifica en ocho campos amplios, que son los aquí referidos: Artes y Humanidades; Ciencias Sociales, Administración y Derecho; Ciencias Naturales, Exactas y de la Computación; Ingeniería, Manufactura y Construcción; Agronomía y Veterinaria, y Salud. La UMSNH no cuenta aún con egresados de las áreas de Educación y servicios.

[3] El primer momento, es cuando laboran antes de egresar

[4] La diferencia entre trabajador independiente y empleado, es que el empleado tiene un patrón de quien depender para hacer su trabajo y el independiente puede prestar sus servicios y se le paga por los insumos que consume y por el servicio que presta.

\section{BY-NC-ND}

